\title{
Objective Predictive Score as a Feasible Biomarker for Short-term Survival in TerminalIy Ill Patients with Cancer
}

\author{
YOSHINAGA OKUGAWA ${ }^{1,2,3 *}$, YUMIKO SHIRAI ${ }^{4 *}$, HIROKO NODONO ${ }^{5}$, FUKUMI MATSUTANI ${ }^{5}$, \\ MARI ITOH $^{5}$, ASAHI HISHIDA ${ }^{6}$, YUKI MORIMOTO ${ }^{2}$, RYUTARO NISHIKAWA ${ }^{2}$, TAKESHI YOKOE ${ }^{2}$, \\ KOJI TANAKA ${ }^{2,3}$, HISASHI URATA ${ }^{2}$, YUJI TOIYAMA ${ }^{3}$, TOSHIMITSU ARAKI ${ }^{3}$, \\ YASUHIRO INOUE ${ }^{3}$, MOTOYOSHI TANAKA ${ }^{2}$, MASATO KUSUNOKI ${ }^{3}$ and CHIKAO MIKI ${ }^{1}$ \\ Departments of ${ }^{1}$ Surgery, ${ }^{2}$ Medical Oncology, ${ }^{4}$ Nutrition and ${ }^{5}$ Nursing, Iga City General Hospital, Mie, Japan; \\ ${ }^{3}$ Department of Gastrointestinal and Pediatric Surgery, Division of Reparative Medicine, \\ Institute of Life Sciences, Mie University Graduate School of Medicine, Mie, Japan; \\ ${ }^{6}$ Department of Preventive Medicine, Nagoya University Graduate School of Medicine, Nagoya, Japan
}

\begin{abstract}
Background: In palliative care, prediction of life expectancy is one of the most crucial issues for patients, family and medical staff, in order to provide appropriate endof-life care. The aim of this study was to formulate a new objective score to predict life expectancy within 1 week for terminally ill patients with cancer. Patients and Methods: Medical records were obtained from 187 terminally-ill patients with cancer who were admitted for palliative care. The biomarkers for a potential 'Objective Predictive Score' were assessed. Results: Profiling of blood parameters demonstrated that elevated levels of alanine aminotransferase $(A L T)$, total bilirubin (T-bil), blood urea nitrogen (BUN), creatinine $(\mathrm{Cr})$ and a decreased platelet count were significantly correlated with death within 1 week in a training cohort. Our formulated Objective Predictive Score was able to predict death within 1 week with high accuracy in a training and a validation cohort. Conclusion: Our scoring system might enable the assessment of prognostication with higher accuracy in a terminal care setting.
\end{abstract}

An accurate prediction for survival of patients with cancer is essential for clinical decision-making $(1,2)$. In the palliative care setting, prediction of life expectancy is one of the most

\footnotetext{
*These Authors contributed equally to this study.

Correspondence to: Yoshinaga Okugawa MD, Ph.D. Department of Surgery, Iga City General Hospital, 831, Shizuku-cho, Iga, Mie 5180823, Japan. Tel: +81595241111, e-mail: yoshinaga.okugawa@ gmail.com; or Chikao Miki MD, Ph.D. Department of Surgery, Iga City General Hospital, 831, Shizuku-cho, Iga, Mie 518-0823, Japan. Tel: +81 595241111, e-mail: harborne3030@gmail.com

Key Words: Palliative care, advanced cancer, prognosis, predictive biomarker, objective predictive scoring system.
}

crucial issues for patients, family and medical staff to enable them to grant final wishes of the patients and to provide appropriate end-of-life care (3-7). With this in mind, various types of prognostic scoring systems have been developed in the past decade.

In these scoring systems, the Palliative Prognostic Index (PPI) is worldwide one of the most widely used to predict life expectancy in terminally ill patients with cancer (8). The PPI consists of scoring of five clinical features (palliative performance status, dyspnoea, oral intake, oedema and delirium). The advantages of this index are its simplicity, wide usability in multiple types of cancer and noninvasiveness for the assessment of prognostication. Several studies have successfully validated the PPI in various hospice settings, with acceptable sensitivity and specificity for end-of-life life expectancy predictions (9-14). However, this prognostic index is still subjective and might be influenced by the experience, psychology and training of the individual palliative physician (15). Recently, Cheng and colleagues evaluated the potential of PPI for the prediction of life expectancy using clinical information from 623 patients with advanced cancer undergoing palliative care (12). The results of this study successfully validated the previous findings of PPI studies and demonstrated that assessment of PPI was able to identify patients who would die within 21 days, with a relatively high area under the receiver operator curve (AUC) value of 0.68 . However, this study also revealed that the PPI score remains overly pessimistic in predicting survival of less than 1 week and overly optimistic in predicting survival over a longer duration (12). Another research group also demonstrated a similar finding (11), and this evidence highlights the urgent need for a more accurate and objective scoring system for predicting life expectancy in patients with advanced cancer undergoing palliative care. 
The prediction of short-term survival (within 1 week) is especially important to be able to fulfil the desire of hospitalised terminally ill patients to die at home. Furthermore, goals of patient management need to shift and a clinical pathway has been developed to guide patient care (16). Accumulating evidence has demonstrated the efficacy of various prognostication models in patients undergoing terminal care, however, almost all of these models predict prognosis based on long-term survival and the predictive model for short-term survival remains to be developed.

Previous work from our group has shown that various types of biochemical data can be used as biomarkers for diagnosis, prognosis and metastasis prediction in patients with gastrointestinal cancer (17-23). The aim of the current study was to develop an objective calculation model using laboratory data to enable prediction of death within 1 week in palliatively treated patients with cancer, in order to improve prediction for short-term survival, and to facilitate provision of high-quality end-of-life care.

\section{Patients and Methods}

Patients and sample collection. This study included total 187 patients (123 men and 64 women) who were admitted to Iga City General Hospital, Japan, for palliative care for advanced malignant disease from two different patient cohorts, as described in Table I. This retrospective study included a discovery cohort, followed by a subsequent clinical validation cohort. In the training cohort, 97 consecutive patients ( 68 men and 29 women) who were admitted to our hospital for palliative care from June 2015 to June 2016 were enrolled. Subsequently, 90 consecutive patients $(55$ men and 35 women) who were hospitalised in our facilities from October 2013 to May 2015 were included in the validation cohort. Inclusion criteria for further analysis were as follows: age $>20$ years; clinical diagnosis of advanced cancer (radiological/histological/cytological confirmation); inpatient status at a palliative care unit (cancer centre or hospice); died in our hospital because of advanced malignant disease; and available electronic medical records (including treatment information). Exclusion criteria were pre-existing complications that might influence survival, such as severe or uncontrollable diabetes mellitus, renal dysfunction and heart disease; haematological malignancies; administration of anticancer therapy including cytotoxic agents, molecular targeting drugs, anti-hormonal therapy, radical surgery and radical radiotherapy; and absence of laboratory examinations during hospitalisation. No patient received chemotherapy at least 2 months prior to the final blood-based evaluation. The mean follow-up duration from the final blood-based evaluation to death was 8.1 days (range $=0-44$ days). The institutional Ethical Review Boards of participating centres approved the study (Approval number: Ibyousou417). This project was a retrospective observational study, therefore whether the investigators obtained individual informed consent from each patient or not depended on the decision of individual Institutional Review Boards.

Data collection. The following data were collected: gender, age, primary cancer site, PPI score, laboratory examination and overall survival (OS). Laboratory examination using blood specimens included levels of albumin, alanine aminotransferase (ALT), aspartate aminotransferase (AST), total bilirubin (T-Bil), blood urea nitrogen (BUN), creatinine $(\mathrm{Cr}), \mathrm{C}$-reactive protein (CRP), haemoglobin $(\mathrm{Hb})$, sodium and potassium, and haematocrit $(\mathrm{Ht})$, neutrophil, lymphocyte and platelet counts. The neutrophillymphocyte ratio (NLR) and platelet-lymphocyte ratio (PLR) were calculated. Onodera-prognostic nutritional index (O-PNI) was calculated based on the serum albumin and total lymphocyte count, using the following equation: $\mathrm{O}-\mathrm{PNI}=10 \times[$ serum albumin $(\mathrm{g} / \mathrm{dl})+$ $0.005 \times$ total lymphocyte count $(\mathrm{n} / \mu)]$. PPI sum scores range from 0 to 15 points based on scoring for the palliative performance scale (PPS) (24) and four additional clinical variables: presence or absence of oedema, delirium, oral intake and resting dyspnoea (8).

Combined objective predictive scoring system. In order to develop a blood-based palliative scoring system, Cox proportional hazard regression models were fitted to the five top risk factors with high area under the receiver operator curve (AUC) values from laboratory biochemical data for predicting death within 1 week: ALT, BUN, creatinine, T-bil, and platelet count. Cox proportional hazard regression models produced (HRs) and based on these data, a linear function was constructed to produce the Objective Scoring System for predicting death within 1 week in a palliative care setting. For the training cohort participants, the Objective Predictive Score was defined using the following steps: $x=-0.49644+[0.018553 \times$ ALT $(\mathrm{U} / \mathrm{l})]+[-0.0090712 \times \mathrm{T}-\mathrm{Bil}(\mathrm{mg} / \mathrm{dl})]+[0.019363 \times \mathrm{BUN}(\mathrm{mg} / \mathrm{dl})]+$ $[0.55853 \times$ creatinine $(\mathrm{mg} / \mathrm{dl})]+[-0.0049938 \times$ platelet count (number/ $\left.\left.\mu 1 \times 10^{3}\right)\right] ; y=\exp (x)$; and Objective Predictive Score $=[y /(1+y)]$, as described previously (25). Based on these data, we validated the accuracy of this scoring system using the validation cohort.

Statistical analysis. Results are expressed as the mean \pm standard deviation and all statistical analyses were performed using Medcalc version 16.4.3 (MedCalc Software, Mariakerke, Belgium). Differences between groups were estimated using the $\chi^{2}$ test and one-way ANOVA, as appropriate. F-Tests were used to assess the equality of variance for comparable groups.

Receiver operating characteristic (ROC) analysis was performed to determine the predictive performance of laboratory biochemical data in distinguishing between patients who died within 1 week from patients who survived more than 1 week. Sensitivity against $100 \%$ minus specificity was plotted at each cut-off threshold and the AUC values that reflected the probability of correctly identifying short- versus longer-term survival were calculated. The optimal cutoff thresholds for predicting death within 1 week were obtained using the Youden index (26). In brief, the optimal cut-off threshold values were determined at the point on the ROC curve at which Youden's index [sensitivity $+(100 \%$ - specificity $)$ ] was maximal. By using these optimal cut-off values, sensitivity, specificity and positive and negative predictive values were calculated. Predictive accuracy was determined by measuring AUC, specificity and sensitivity. A predictive model with an AUC of more than 0.7 was considered to be sufficiently discriminative; an AUC of 0.5 is equivalent to a 'coin toss' $(27,28)$. For time-to-event analyses, survival estimates were calculated using Kaplan-Meier analysis and groups were compared with the log-rank test. Overall survival (OS) was measured from the date of the final blood-based laboratory examination to the date of death, resulting from any cause. For the assessment of the performance of prognostic markers for OS, power calculations were based on the detection difference of 0.05 between 
Table I. Demographic characteristics of all patients from each palliative cohort.

\begin{tabular}{lccc}
\hline Characteristic & $\begin{array}{c}\text { Training cohort } \\
\mathrm{n}=97\end{array}$ & $\begin{array}{c}\text { Validation cohort } \\
\mathrm{n}=90\end{array}$ & $p$-Value \\
\hline Gender & & & \\
Male & 68 & 57 & $0.33^{\#}$ \\
Female & 29 & 33 & \\
Age (years) & & & \\
Mean (SD) & $75.6(11.4)$ & $74.1(10.0)$ & $0.43^{*}$ \\
Median (range) & $78(47-99)$ & $74(47-97)$ & \\
Primary tumour site & & & \\
Gastrointestinal tract & 45 & 44 & $0.94^{\# \#}$ \\
Hepatobiliary & 9 & 7 & \\
Pancreas & 8 & 10 & \\
Lung & 12 & 11 & \\
Breast & 2 & 6 & \\
Genitourinary & 16 & 8 & \\
Other & 5 & 4 & \\
Survival & & & \\
Mean (SD) & $7.4(8.8)$ & $8.7(9.3)$ & $0.33^{*}$ \\
Median (range) & $4(0-44)$ & $5.5(0-39)$ & \\
\hline
\end{tabular}

aFrom day of final evaluation. ${ }^{\#}$ Chi-square test, ${ }^{\# \#}$ Chi-square test for trend, *one-way ANOVA.

favourable and unfavourable prognostic groups. We estimated 32 and 50 patients (distributed 7:11 between the two groups) were needed to achieve $95 \%$ power in order to substantiate more than $15 \%$ and $50 \%$ differences in prognostic and recurrent outcomes, respectively, at a significance level of 0.05 , using a two-sided logrank test. The Cox proportional hazards model was used to estimate HRs for death. ROC curves were established to determine the cutoff values for analysis of prognosis by Youden's index. Assumption of proportionality was confirmed for the Cox proportional hazard analyses by generating Kaplan-Meier survival curves (e.g. high vs. low scoring groups) and by ensuring that the two curves did not intersect. Multivariate logistic regression models were used to predict factors indicative of death within 1 week in patients undergoing palliative care. Forced-entry regression was used to include these variables in all multivariable equations to analyze whether each of the predictors affected the outcome, after adjusting for known confounders. All $p$-values were two-sided and those less than 0.05 were considered statistically significant.

\section{Results}

Patient demographics. Demographic data for the patients are shown in Table I. The mean age was 75.6 years (range 47-99 years) in the training cohort and 74.1 years (range 4797 years) in the validation cohort. There were no significant differences in any of these factors between cohorts.

Biochemical factors predicting 1 week life expectancy in advanced cancer patients in the training cohort. In order to determine which biochemical parameters were able to predict life expectancy in patients with advanced cancer undergoing
Table II. Accuracy of objective and subjective data for prediction of death within 1 week in the training cohort.

\begin{tabular}{|c|c|c|c|c|c|}
\hline \multirow[t]{2}{*}{ Variable } & \multirow[t]{2}{*}{ Subgroup $\#$} & \multicolumn{3}{|c|}{ Death within 1-week } & \multirow[t]{2}{*}{$p$-Value } \\
\hline & & Sensitivity & Specificity & AUC & \\
\hline Albumin & $\begin{array}{l}<2.5 \mathrm{~g} / \mathrm{dl} \\
\geq 2.5 \mathrm{~g} / \mathrm{dl}\end{array}$ & 28.8 & 93.6 & 0.58 & 0.17 \\
\hline AST & $\begin{array}{l}>42 \mathrm{u} / 1 \\
\leq 42 \mathrm{u} / 1\end{array}$ & 63.6 & 67.7 & 0.65 & 0.008 \\
\hline ALT & $\begin{array}{l}>45 \mathrm{u} / 1 \\
\leq 45 \mathrm{u} / 1\end{array}$ & 47 & 87.1 & 0.68 & 0.0008 \\
\hline T-Bil & $\begin{array}{l}>0.56 \mathrm{mg} / \mathrm{dl} \\
\leq 0.56 \mathrm{mg} / \mathrm{dl}\end{array}$ & 75.8 & 61.3 & 0.67 & 0.004 \\
\hline BUN & $\begin{array}{l}>39.5 \mathrm{mg} / \mathrm{dl} \\
\leq 39.5 \mathrm{mg} / \mathrm{dl}\end{array}$ & 60.6 & 80.7 & 0.78 & $<0.0001$ \\
\hline $\mathrm{Cr}$ & $\begin{array}{l}>1.39 \mathrm{mg} / \mathrm{dl} \\
\leq 1.39 \mathrm{mg} / \mathrm{dl}\end{array}$ & 53 & 83.9 & 0.71 & 0.0001 \\
\hline CRP & $\begin{array}{l}>2.26 \mathrm{mg} / \mathrm{dl} \\
\leq 2.26 \mathrm{mg} / \mathrm{dl}\end{array}$ & 89.2 & 32.3 & 0.58 & 0.21 \\
\hline $\mathrm{Hb}$ & $\begin{array}{l}>7.4 \mathrm{~g} / \mathrm{dl} \\
\leq 7.4 \mathrm{~g} / \mathrm{dl}\end{array}$ & 84.9 & 35.5 & 0.62 & 0.045 \\
\hline $\mathrm{Ht}$ & $\begin{array}{l}>35.3 \% \\
\leq 35.3 \%\end{array}$ & 33.3 & 87.1 & 0.61 & 0.06 \\
\hline Plt & $\begin{array}{l}\leq 21.8 \quad 10^{4} / \mathrm{ul} \\
>21.8 \quad 10^{4} / \mathrm{ul}\end{array}$ & 75.8 & 64.5 & 0.72 & 0.0002 \\
\hline NLR & $\begin{array}{l}>9.6 \\
\leq 9.6\end{array}$ & 78 & 51.9 & 0.66 & 0.008 \\
\hline PLR & $\begin{array}{l}\leq 389.2 \\
>389.2\end{array}$ & 81.4 & 40.7 & 0.6 & 0.14 \\
\hline PNI & $\begin{array}{l}>28.2 \\
\leq 28.2\end{array}$ & 40.7 & 85.2 & 0.55 & 0.48 \\
\hline Sodium & $\begin{array}{l}>142 \mathrm{mmol} / 1 \\
\leq 142 \mathrm{mmol} / \mathrm{l}\end{array}$ & 16.7 & 100 & 0.52 & 0.77 \\
\hline Potassium & $\begin{array}{l}>4.8 \mathrm{mmol} / 1 \\
\leq 4.8 \mathrm{mmol} / 1\end{array}$ & 57.6 & 74.2 & 0.65 & 0.012 \\
\hline PPI & $\begin{array}{l}>7 \\
\leq 7\end{array}$ & 71.4 & 71.4 & 0.66 & 0.016 \\
\hline OPS & $\begin{array}{l}>0.735 \\
\leq 0.735\end{array}$ & 65.2 & 90.3 & 0.84 & $<0.0001$ \\
\hline
\end{tabular}

AUC: Area under the receiver operator curve, AST: aspartate aminotransferase, ALT: alanine aminotransferase, T-Bil: total bilirubin, BUN: blood urea nitrogen, $\mathrm{Cr}$ : creatinine, CRP: C-reactive protein, $\mathrm{Hb}$ : haemoglobin, Ht: haematocrit, Plt: Platelet, NLR: neutrophil to lymphocyte ratio, OPS: Objective predictive score, PLR: platelet to lymphocyte ratio, PNI: Prognostic Nutritional Index (Onodra), PPI: Palliative Prognostic Index. \#Determined by Receiver operating characteristic analysis with Youden's index, for death within 1 week in patients undergoing palliative care.

palliative care, we analysed the accuracy of each biochemical factor to predict death within 1 week in the training cohort (Table II). Profiling of each biochemical factor revealed that elevated levels of AST, ALT, T-bil, BUN, creatinine, $\mathrm{Hb}, \mathrm{NLR}$ and potassium and a decreased platelet count were significantly correlated with death within 1 week. These factors, particularly the top five factors with high 
AUC, namely ALT, T-bil, BUN, creatinine, and platelet count, were able to predict death with an accuracy of more than 0.67 , based on ROC curve analysis ( $\mathrm{AUC}=0.68$, $p=0.0008 ; \mathrm{AUC}=0.67, p=0.004 ; \mathrm{AUC}=0.78, p<0.0001$; $\mathrm{AUC}=0.71, p=0.0001 ; \mathrm{AUC}=0.72, p=0.0002$, respectively, Table II and Figure 1).

Combined Objective Predictive Score successfully identified patients who would die within 1 week. Based on these data, we progressed to make the calculation model, called the Objective Predictive Score, in order to assess the degree of life expectancy using these factors. The Objective Predictive Score correctly identified patients with advanced cancer with a life expectancy of less than 1 week with high accuracy in the training cohort (sensitivity $=65.2 \%$; specificity $=90.3 \%$; AUC $=0.84 ; p<0.0001$, Table II and Figure 1).

The Objective Predictive Score was significantly correlated with prognosis. Subsequently, time-to-event analysis was used to evaluate the prognostic relevance of the Objective Predictive Score for survival in the training cohort. The cutoff threshold for the Objective Predictive Score for this analysis was determined with Youden's index from the ROC curves in the training cohort. A high Objective Predictive Score was significantly correlated with poor prognosis for survival, compared to the low-scoring group in the training cohort $(p<0.0001$, Figure $2 \mathrm{a})$. Furthermore, we validated the potential of this scoring system using clinical data from the validation cohort and the same cut-off value as for the training cohort. This analysis clearly revealed that a high Objective Predictive Score was significantly correlated with poor prognosis and survival, compared to a low score, in the validation cohort $(p=0.0063$; Figure $2 b)$, as well as the total patient cohort $(p<0.0001$; Figure $2 c)$. In order to determine the value of a high Objective Predictive Score as a predictive biomarker for prognosis in patients with advanced cancer, we performed multivariate Cox regression analysis. The data revealed that a high Objective Predictive Score was an independent prognostic factor for poor survival $[\mathrm{HR}=2.75$, $95 \%$ confidence interval $(\mathrm{CI})=1.7-4.46, p<0.0001)$ in patients undergoing palliative care for advanced cancer from the training cohort (Table III). In addition, a high Objective Predictive Score was also an independent prognostic factor for survival in both the validation and total cohorts $(\mathrm{HR}=1.85$, $95 \% \mathrm{CI}=1.18-2.9, p=0.0008 ; \mathrm{HR}=2.16,95 \% \mathrm{CI}=1.58-2.94$, $p<0.0001$, respectively, Table III).

Objective predictive score successfully predicted lifeexpectancy of less than 1 week in a validation cohort. Finally, we evaluated accuracy of the Objective Predictive Score for prediction of life expectancy less than 1 week using the validation cohort. The score was successfully validated and showed that a high score was able to identify those patients who would die within 1 week, with high accuracy (sensitivity $=63.0 \%$, specificity $=80.6 \%$, AUC $=0.72$, Figure $3 a)$. Consistent with these data, assessment of the Objective Predictive Score differed for patients with and those without life expectancy of less than 1 week in the total cohort (sensitivity $=65.8 \%$, specificity $=77.6 \%$, AUC $=0.78$, Figure $3 \mathrm{~b}$ ). Based on these findings, we performed multivariate logistic analysis to determine the clinical significance of the Objective Predictive Score as a predictive biomarker for death within 1 week in a palliative care setting. Notably, a high Objective Predictive Score was shown to be an independent predictive factor for life expectancy of less than 1 week in the training cohort [odds ratio $(\mathrm{OR}=16,95 \%$ $\mathrm{CI}=4.18-61.5, p=0.0001$, validation cohort $(\mathrm{OR}=4.06,95 \%$ $\mathrm{CI}=1.62-10.2, p=0.0027)$ and total cohort $(\mathrm{OR}=5.82,95 \%$ $\mathrm{CI}=2.95-11.5, p<0.0001$ ) (Table IV)], adjusted for gender, age and disease type. Furthermore, combining the Objective Predictive Score with the well-established PPI showed a high accuracy for predicting life-expectancy of less than 1 week in terminally ill patients with cancer (AUC $=0.83$, sensitivity $=67.9 \%$, specificity $=89.3 \%$, Figure 4 ). Collectively, these data, highlight the clinical feasibility of a predictive scoring system for life expectancy of less than 1 week in patients with advanced cancer undergoing palliative care.

\section{Discussion}

Prediction of life expectancy is one of the most important issues for patients with terminal cancer in palliative care (29, 30). Accurate knowledge of prognosis often helps patients themselves in their preparation for dying (3-5), as well as families in fulfilling patients' final wishes (6) and medical personnel in providing appropriate end-of-life care (7). Although accumulating evidence suggests the importance of various types of prognostication systems for patients with terminal cancer, the clinical feasibility of prognostication systems using biochemical parameters remains unclear.

In this study, we systematically investigated the potential role of biochemical parameters for prognostication in patients with terminal cancer. Firstly, parameters of several biochemical factors, including ALT, T-bil, BUN, creatinine, and platelet count were able to predict death within 1 week with an accuracy of more than 0.67 , based on ROC curve analysis. Secondly, a prognostication formula using these five factors combined, namely the Objective Predictive Score, improved the identification of patients with a life expectancy of 1 week or less. Furthermore, multivariate Cox regression analysis revealed that a high Objective Predictive Score was an independent risk factor for OS. Finally, these results were confirmed in a validation cohort and ROC analysis demonstrated that a high Objective Predictive Score successfully validated prediction for life expectancy of less than 1 week in a validation cohort. 
a

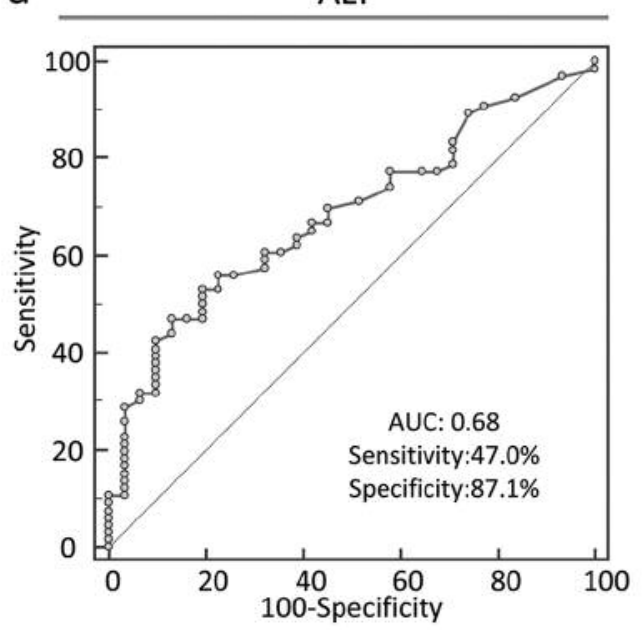

C

BUN

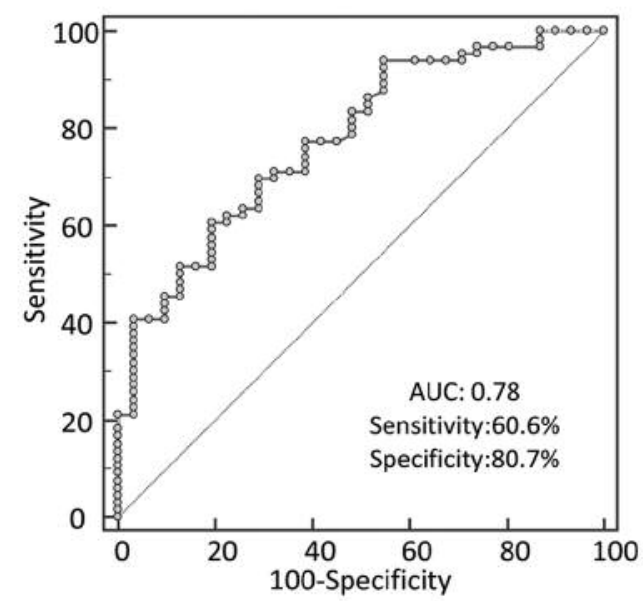

$\mathrm{e}$

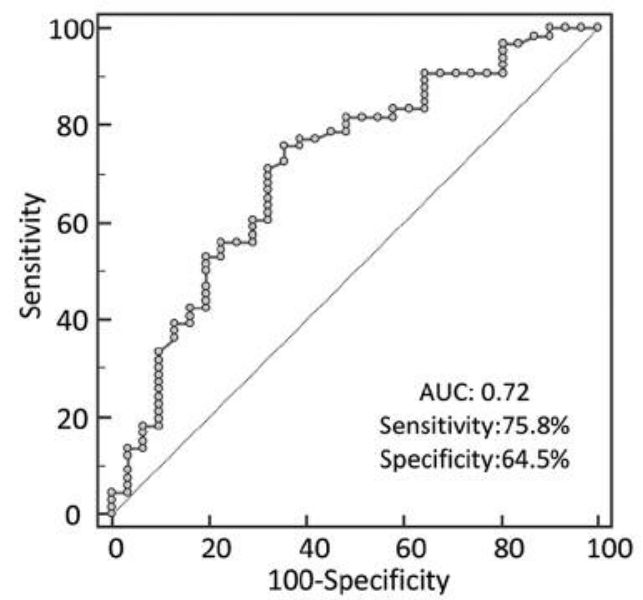

b T-bil

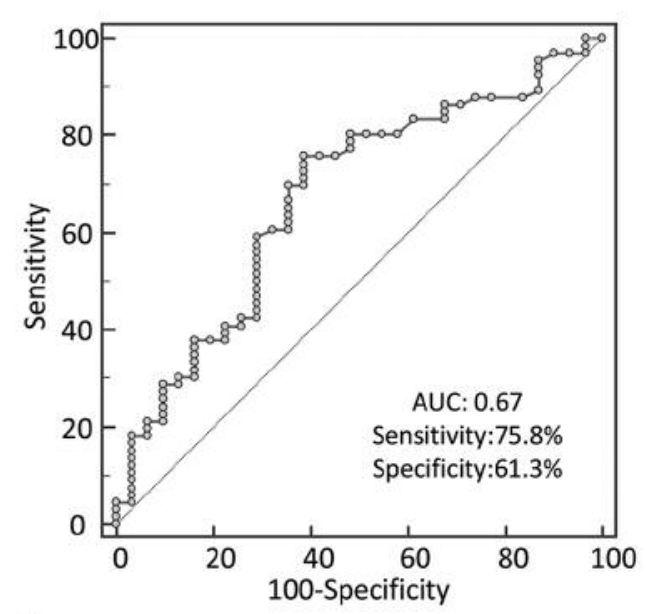

d

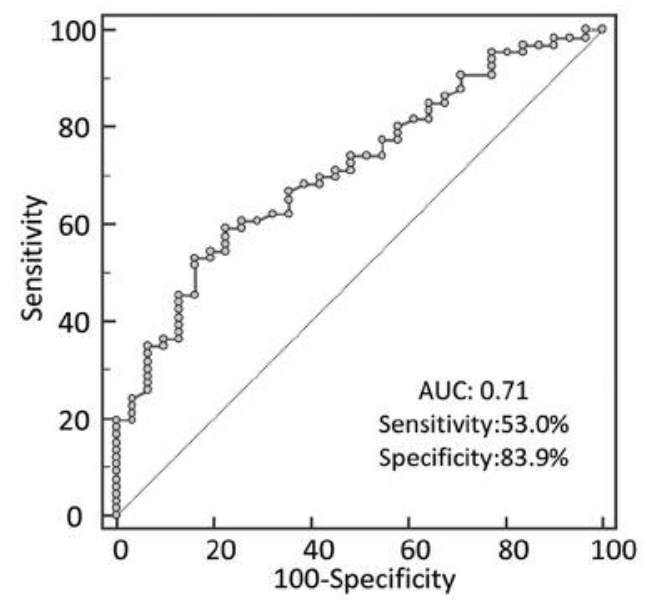

f

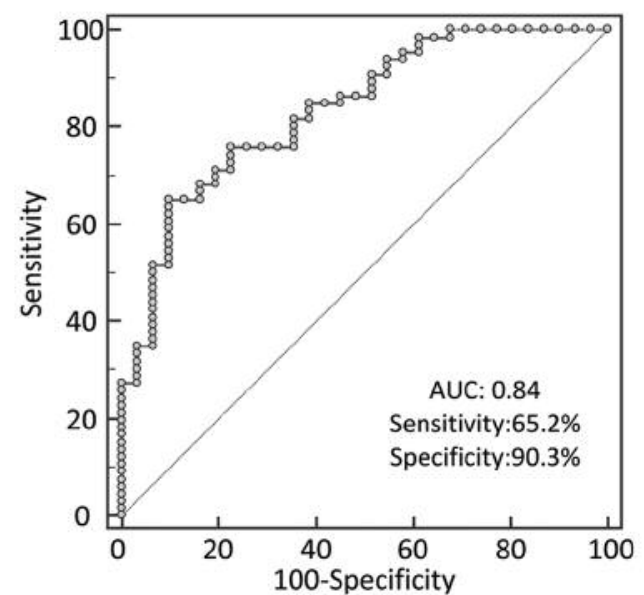

Figure 1. Receiver operating characteristic curve analysis for alanine aminotransferase (ALT) (a), total bilirubin (T-bil) (b), blood urea nitrogen $(B U N)(c)$, creatinine $(C r)(d)$ and platelet count $(e)$, and the combined Objective Predictive Score $(f)$ in identifying patients with a life expectancy of less than 1 week. These gave predictive values for life expectancy of less than 1 week with a high accuracy of more than 0.67 based on ROC curve analysis in a training cohort. AUC: Area under the receiver operator curve. 


\section{a}
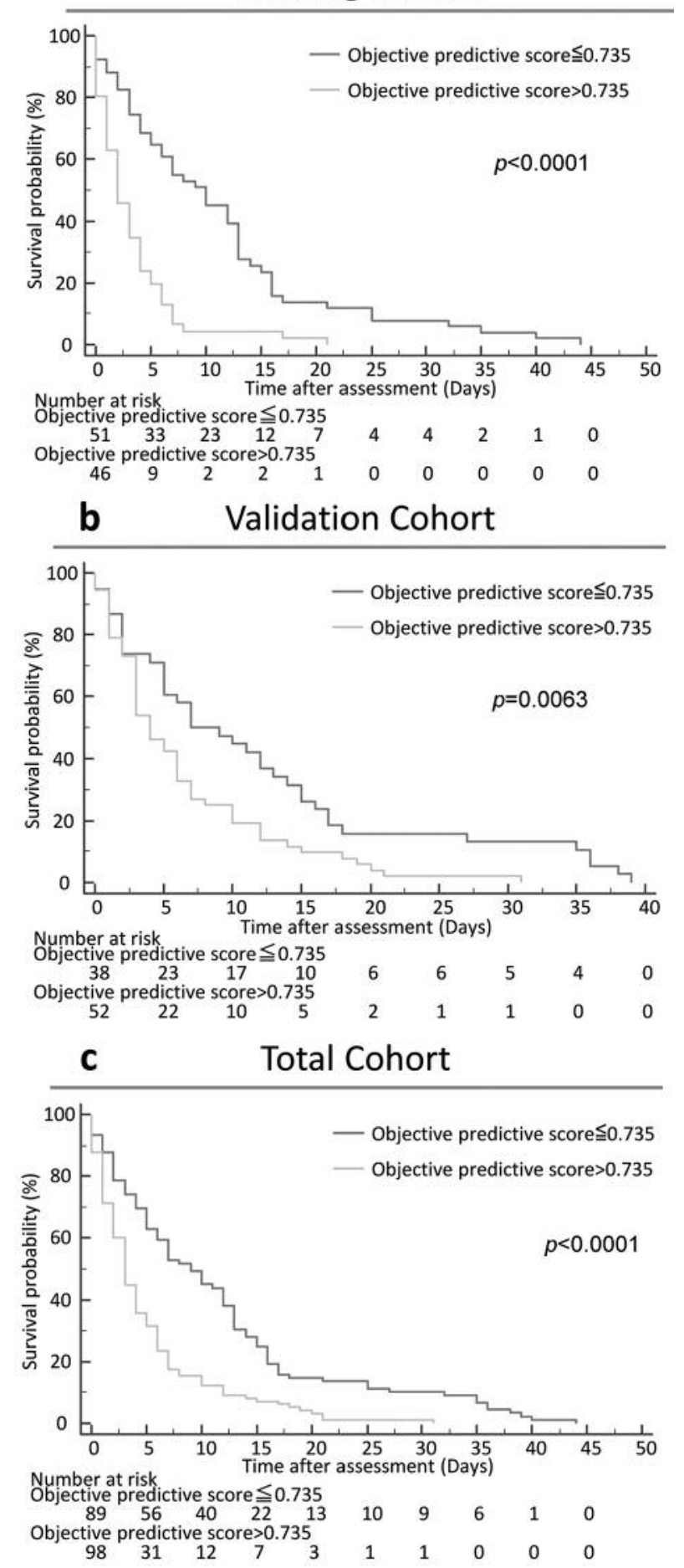

Figure 2. Prognostic value of the Objective Predictive Score (OPS) in overall survival $(O S)$ of terminally ill patients with cancer. KaplanMeier survival curves were plotted for OS of terminally cancer patients based on the Objective Predictive Score using a cut-off of 0.735. An Objective Predictive Score above the cut-off was significantly associated with poor survival in the training $(a)$, validation $(b)$ and total (combined) (c) cohorts by log-rank test.
In palliative care for patients with terminal malignant disease, one of the major concerns is an accurate prediction for the probability of long- and short-term survival. Especially for short-term survival, clinicians need to change patient management in order to guide care (16). Although patients with an expected prognosis of a couple of weeks are generally incapable of oral fluid intake because of progressive cachexia, simple hydration at $1000-1500 \mathrm{ml} /$ day is not recommended to improve the general quality of life (31). Several scoring systems for prognostication in terminally ill patients with cancer have been developed in the past decade $(8,14,32)$. However, most systems, including palliative prognosis score and palliative prognostic index, use subjective clinical symptoms, including presence of dyspnoea, anorexia and oedema (8) and diagnosis can be influenced by the clinical skills of the individual medical staff. Furthermore, these scoring systems predict long-term life expectancy less than a couple of months, and not to be appropriate for prediction of life expectancy less than one-week in hospice care. In general, clinical prognostication is dependent on intuition, experience and changes in clinical condition, in particular systemic disability over a period of time. In order to provide high quality end-of-life care for terminally ill patients and their families, it is important to have a common recognition of prognostication for all medical staff. In contrast, prediction for life expectancy, especially in later stages of the illness, is difficult for all clinical staff (33), therefore more accurate and objective scoring systems are urgently needed.

Several studies have demonstrated various types of scoring systems for short-term prognostication in terminally ill patients with cancer. Chiang and colleagues formulated a prognostic scale using eight clinical factors including Eastern Cooperative Oncology Group (ECOG) performance status, weight loss, cognitive impairment, general fatigue, presence of oedema, ascites and lung and liver metastases, for prediction of life expectancy of less than 1 week (32). This prognostic scale was able to discriminate patients with and those without a life expectancy of 1 week, with comparatively high accuracy in a training cohort and a testing cohort $(\mathrm{AUC}=0.72$ and 0.66 , respectively) (34). The same study also evaluated clinical data from 727 patients with terminal cancer to formulate a prognostication score for prediction of prognostic 7-day survival and identified five clinical and biochemical factors, namely cognitive status, oedema, ECOG performance status, BUN and respiratory rate, as independent risk factors for life expectancy less than 1 week. Based on these data, they constructed a formula for a predictive model using these five factors and successfully validated the prediction of death within 1 week, with relatively high sensitivity and specificity (sensitivity $=71.0 \%$, specificity $=57.7 \%$ ) (32). Consistent with these previous studies, our study demonstrated that several blood parameters, including BUN, creatinine, ALT, T-bil and platelet count, were able to identify patients with terminal 
Table III. Multivariate analysis for predictors of overall survival in the training, validation and total (combined) cohorts.

\begin{tabular}{|c|c|c|c|c|c|c|}
\hline \multirow[t]{2}{*}{ Variable } & \multicolumn{3}{|c|}{ Univariate } & \multicolumn{3}{|c|}{ Multivariate } \\
\hline & HR & $95 \% \mathrm{CI}$ & $p$-Value & HR & $95 \% \mathrm{CI}$ & $p$-Value \\
\hline \multicolumn{7}{|l|}{ Training cohort } \\
\hline Gender (male) & 1.6 & $1.02-2.5$ & 0.041 & 1.41 & $0.88-2.22$ & 0.15 \\
\hline Age $(>78 \text { years })^{\#}$ & 0.7 & $0.46-1.06$ & 0.09 & 0.86 & $0.55-1.33$ & 0.49 \\
\hline Primary tumor site & 0.99 & $0.87-1.12$ & 0.89 & 0.95 & $0.83-1.08$ & 0.43 \\
\hline OPS $(>0.735)^{\dagger}$ & 2.9 & $1.86-4.51$ & $<0.0001$ & 2.75 & $1.7-4.46$ & $<0.0001$ \\
\hline \multicolumn{7}{|l|}{ Validation cohort. } \\
\hline Gender (male) & 1.16 & $0.75-1.8$ & 0.49 & 1.27 & $0.81-1.99$ & 0.3 \\
\hline Age $(>74 \text { years })^{\#}$ & 1.11 & $0.73-1.69$ & 0.62 & 1.11 & $0.73-1.69$ & 0.63 \\
\hline Primary tumor site & 1.01 & $0.89-1.14$ & 0.91 & 1.04 & $0.91-1.18$ & 0.59 \\
\hline OPS $(>0.735)^{\dagger}$ & 1.79 & $1.15-2.79$ & 0.01 & 1.85 & $1.18-2.9$ & 0.0008 \\
\hline \multicolumn{7}{|l|}{ Total cohort } \\
\hline Gender (male) & 1.39 & $1.02-1.89$ & 0.04 & 1.39 & $1.01-1.91$ & $0.04 *$ \\
\hline Age $(>77 \text { years })^{\#}$ & 0.96 & $0.72-1.28$ & 0.76 & 1.06 & $0.79-1.42$ & 0.71 \\
\hline Primary tumor site & 0.99 & $0.92-1.09$ & 0.94 & 1.01 & $0.92-1.1$ & 0.94 \\
\hline OPS $(>0.735)^{\dagger}$ & 2.13 & $1.57-2.9$ & $<0.0001$ & 2.16 & $1.58-2.94$ & $<0.0001$ \\
\hline
\end{tabular}

CI: Confidence interval, HR: hazard ratio, OPS: Objective predictive score. \#Median age at surgery. ${ }^{\dagger}$ Cut-off for combined predictive probability as determined by Receiver operating characteristic analysis with Youden's index, for death within 1 week in patients undergoing palliative care.

Table IV. Multivariate analysis for prediction of death within 1 week in the training, validation and total (combined) cohorts.

\begin{tabular}{|c|c|c|c|c|c|c|}
\hline \multirow[t]{2}{*}{ Variable } & \multicolumn{3}{|c|}{ Univariate } & \multicolumn{3}{|c|}{ Multivariate } \\
\hline & OR & $95 \% \mathrm{CI}$ & $p$-Value & OR & $95 \% \mathrm{CI}$ & $p$-Value \\
\hline \multicolumn{7}{|l|}{ Training cohort } \\
\hline Gender (male) & 2.8 & $1.12-6.97$ & 0.027 & 1.98 & $0.66-5.91$ & 0.22 \\
\hline Age $(>78 \text { years })^{\#}$ & 0.73 & $0.31-1.72$ & 0.47 & 0.82 & $0.29-2.28$ & 0.7 \\
\hline Primary tumor site & 1.08 & $0.82-1.43$ & 0.57 & 0.97 & $0.68-1.39$ & 0.88 \\
\hline OPS $(>0.735)^{\dagger}$ & 17.4 & $4.78-63.6$ & $<0.0001$ & 16 & $4.18-61.5$ & 0.0001 \\
\hline \multicolumn{7}{|l|}{ Validation cohort } \\
\hline Gender (male) & 1.17 & $0.49-2.8$ & 0.72 & 1.45 & $0.55-3.84$ & 0.46 \\
\hline Age $(>74 \text { years })^{\#}$ & 1.12 & $0.48-2.6$ & 0.8 & 1.37 & $0.55-3.43$ & 0.5 \\
\hline Primary tumor site & 1.15 & $0.88-1.49$ & 0.29 & 1.24 & $0.92-1.65$ & 0.15 \\
\hline OPS $(>0.735)^{\dagger}$ & 3.73 & $1.53-9.08$ & 0.0037 & 4.06 & $1.62-10.2$ & 0.0027 \\
\hline \multicolumn{7}{|l|}{ Total cohort } \\
\hline Gender (male) & 1.82 & $0.97-3.4$ & 0.06 & 1.84 & $0.9-3.76$ & 0.09 \\
\hline $\operatorname{Age}(>77 \text { years })^{\#}$ & 0.95 & $0.52-1.73$ & 0.87 & 1.09 & $0.56-2.11$ & 0.81 \\
\hline Primary tumor site & 1.12 & $0.93-1.35$ & 0.25 & 1.14 & $0.92-1.4$ & 0.24 \\
\hline OPS $(>0.735)^{\dagger}$ & 6.11 & $3.13-11.9$ & $<0.0001$ & 5.82 & $2.95-11.5$ & $<0.0001$ \\
\hline
\end{tabular}

CI: Confidence interval, OR:odds ratio, OPS: Objective predictive score. ${ }^{\# T h e ~ m e d i a n ~ a g e ~ a t ~ s u r g e r y . ~}{ }^{\dagger}$ Cut-off for combined predictive probability as determined by Receiver operating characteristic analysis with Youden's index, for death within 1 week in patients undergoing palliative care.

cancer with a life expectancy of less than 1 week. However, previous scoring systems for prediction of life expectancy less than 1 week also includes several subjective parameters, such as ECOG performance status, weight loss, cognitive status, general fatigue, and presence of oedema, and all of parameters in our Objective Predictive Score consisted of biochemical factors using blood test. Based on this standpoint, our Objective Predictive Score could be used more objectively, easy-to-evaluate for all staffs, and easy-to share information in all staffs of palliative care unit to predict life expectancy less than one-week. Furthermore, our Objective Predictive Score was successfully validated using clinical data from a different patient cohort. Collectively, our current findings demonstrate the clinical feasibility of a laboratory-based prognostic scoring system for the prediction of a life expectancy of less than 1 week in patients with terminal cancer. 
a

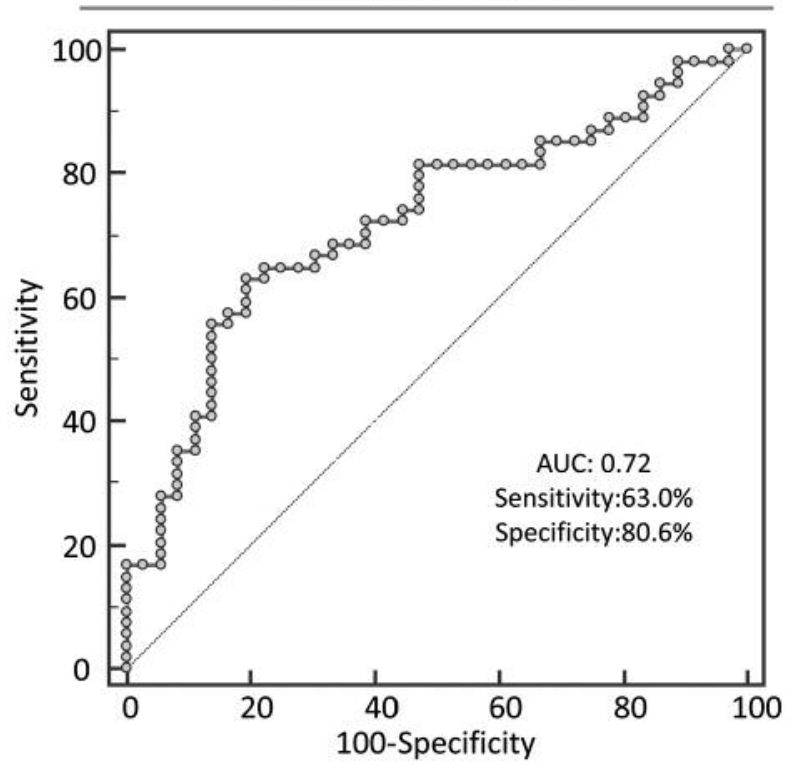

b

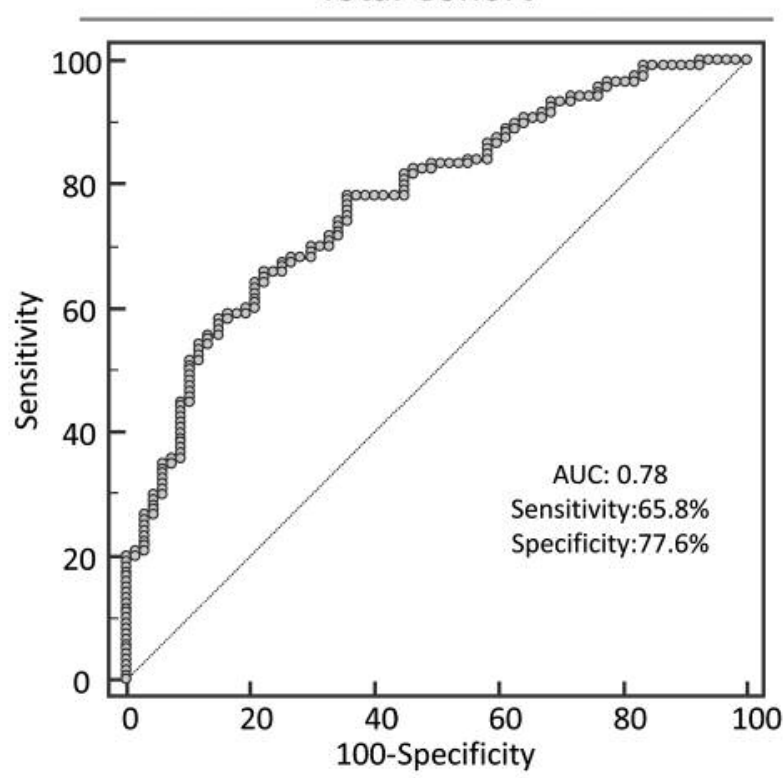

Figure 3. Predictive value of the Objective Predictive Score for death within 1 week in the validation cohort and the total cohort. Receiver operating characteristic analysis curve analysis demonstrated that the Objective Predictive Score was able to identify patients with a life expectancy of less than 1 week, with area under the receiver operator curve (AUC) value of 0.72 in the validation cohort and 0.78 in the total cohort.

In summary, this study provides novel evidence for the clinical significance of our formulated Objective Prognostic Score for prediction of short-term survival in a clinical terminal care setting. Our scoring system might enable high-accuracy

\section{Objective predictive score with PPI}

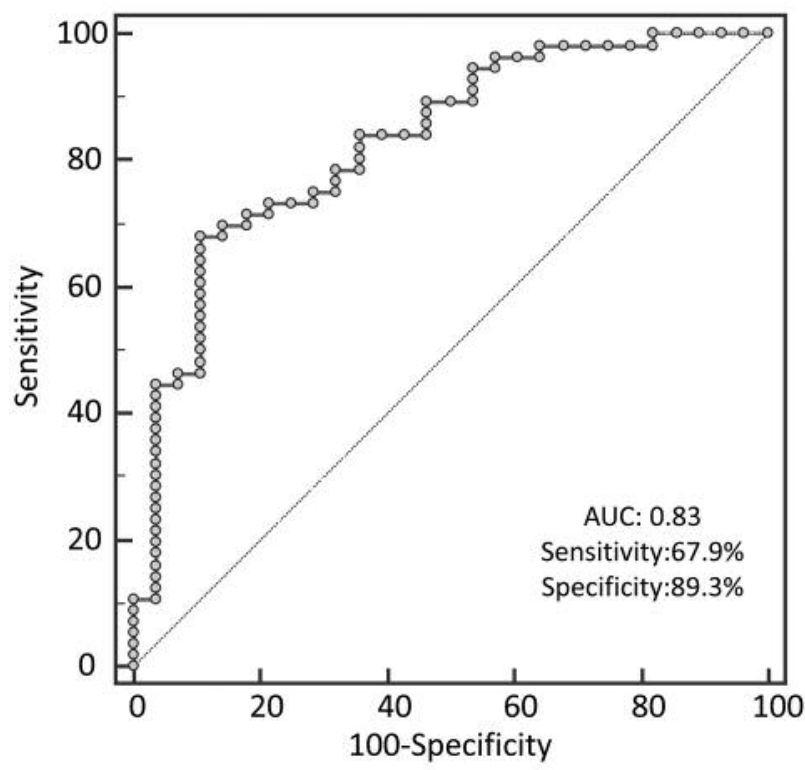

Figure 4. Predictive value of combined Objective Predictive Score with the well-established Palliative Prognostic Index for death within 1 week in terminally ill patients with cancer. Receiver operating characteristic analysis curve analysis for the combined scoring system showed high accuracy for prediction of life expectancy of less than 1 week in terminally ill patients with cancer. AUC: Area under the receiver operator curve.

pre-notification for all medical staff and may allow higherquality end-of-Iife care for patients in a terminal care setting.

\section{Conflicts of Interest}

The Authors have no conflicts of interest to disclose.

\section{References}

1 Hui D, Con A, Christie G and Hawley PH: Goals of care and end-of-life decision making for hospitalized patients at a Canadian tertiary care cancer center. J Pain Symptom Manage 38(6): 871-881, 2009.

2 Hui D: Prognostication of survival in patients with advanced cancer: Predicting the unpredictable? Cancer Control 22(4): 489-497, 2015.

3 Steinhauser KE, Christakis NA, Clipp EC, McNeilly M, Grambow S, Parker J and Tulsky JA: Preparing for the end of life: Preferences of patients, families, physicians, and other care providers. J Pain Symptom Manage 22(3): 727-737, 2001.

4 Alifrangis C, Koizia L, Rozario A, Rodney S, Harrington M, Somerville C, Peplow $\mathrm{T}$ and Waxman $\mathrm{J}$ : The experiences of cancer patients. QJM 104(12): 1075-1081, 2011.

5 Innes S and Payne S: Advanced cancer patients' prognostic information preferences: A review. Palliat Med 23(1): 29-39, 2009.

6 Adams E, Boulton M and Watson E: The information needs of partners and family members of cancer patients: A systematic literature review. Patient Educ Couns 77(2): 179-186, 2009. 
7 Rose JH, O’Toole EE, Dawson NV, Thomas C, Connors AF, Jr., Wenger N, Phillips RS, Hamel MB, Reding DT, Cohen HJ and Lynn J: Generalists and oncologists show similar care practices and outcomes for hospitalized late-stage cancer patients. Support investigators. Study to understand prognoses and preferences for outcomes and risks for treatment. Med Care 38(11): 1103-1118, 2000 .

8 Morita T, Tsunoda J, Inoue S and Chihara S: The palliative prognostic index: A scoring system for survival prediction of terminally ill cancer patients. Support Care Cancer 7(3): 128$133,1999$.

9 Stiel S, Bertram L, Neuhaus S, Nauck F, Ostgathe C, Elsner F and Radbruch L: Evaluation and comparison of two prognostic scores and the physicians' estimate of survival in terminally ill patients. Support Care Cancer 18(1): 43-49, 2010.

10 Stone CA, Tiernan E and Dooley BA: Prospective validation of the palliative prognostic index in patients with cancer. J Pain Symptom Manage 35(6): 617-622, 2008.

11 Maltoni M, Scarpi E, Pittureri C, Martini F, Montanari L, Amaducci E, Derni S, Fabbri L, Rosati M, Amadori D and Nanni O: Prospective comparison of prognostic scores in palliative care cancer populations. Oncologist 17(3): 446-454, 2012.

12 Cheng WH, Kao CY, Hung YS, Su PJ, Hsieh CH, Chen JS, Wang HM and Chou WC: Validation of a palliative prognostic index to predict life expectancy for terminally ill cancer patients in a hospice consultation setting in taiwan. Asian Pac J Cancer Prev 13(6): 2861-2866, 2012.

13 Maltoni M, Caraceni A, Brunelli C, Broeckaert B, Christakis N, Eychmueller S, Glare P, Nabal M, Vigano A, Larkin P, De Conno F, Hanks G and Kaasa S: Prognostic factors in advanced cancer patients: Evidence-based clinical recommendations - a study by the Steering Committee of the European Association for Palliative Care. J Clin Oncol 23(25): 6240-6248, 2005.

14 Lau F, Cloutier-Fisher D, Kuziemsky C, Black F, Downing M, Borycki E and Ho F: A systematic review of prognostic tools for estimating survival time in palliative care. J Palliat Care 23(2): 93-112, 2007.

15 Glare P: Clinical predictors of survival in advanced cancer. J Support Oncol 3(5): 331-339, 2005.

16 Furst CJ and Doyle D: The Terminal Phase. Oxford University Press: Oxford, pp. 1119-1133, 2004.

17 Okugawa Y, Toiyama Y, Mohri Y, Tanaka K, Kawamura M, Hiro J, Araki T, Inoue Y, Miki C and Kusunoki M: Elevated serum concentration of monocyte chemotactic protein $4(\mathrm{MCP}-4)$ as a novel non-invasive prognostic and predictive biomarker for detection of metastasis in colorectal cancer. J Surg Oncol 114(4): 483-489, 2016.

18 Hur K, Toiyama Y, Okugawa Y, Ide S, Imaoka H, Boland CR and Goel A: Circulating microrna-203 predicts prognosis and metastasis in human colorectal cancer. Gut doi:10.1136/gutjnl2014-308737, 2015.

19 Toiyama Y, Tanaka K, Kitajima T, Shimura T, Imaoka H, Mori K, Okigami M, Yasuda H, Okugawa Y, Saigusa S, Ohi M, Inoue Y, Mohri Y, Goel A and Kusunoki M: Serum angiopoietin-like protein 2 as a potential biomarker for diagnosis, early recurrence and prognosis in gastric cancer patients. Carcinogenesis 36(12): 1474-1483, 2015.

20 Toiyama Y, Inoue Y, Kawamura M, Kawamoto A, Okugawa Y, Hiro J, Saigusa S, Tanaka K, Mohri Y and Kusunoki M: Elevated platelet count as predictor of recurrence in rectal cancer patients undergoing preoperative chemoradiotherapy followed by surgery. Int Surg 100(2): 199-207, 2015.

21 Hur K, Toiyama Y, Schetter AJ, Okugawa Y, Harris CC, Boland $\mathrm{CR}$ and Goel A: Identification of a metastasis-specific microrna signature in human colorectal cancer. J Natl Cancer Inst 107(3): 10.1093/jnci/dju492, 2015.

22 Toiyama Y, Tanaka K, Kitajima T, Shimura T, Kawamura M, Kawamoto A, Okugawa Y, Saigusa S, Hiro J, Inoue Y, Mohri Y, Goel A and Kusunoki M: Elevated serum angiopoietin-like protein 2 correlates with the metastatic properties of colorectal cancer: A serum biomarker for early diagnosis and recurrence. Clin Cancer Res 20(23): 6175-6186, 2014.

23 Saigusa S, Tanaka K, Inoue Y, Toiyama Y, Okugawa Y, Iwata T, Mohri Y and Kusunoki M: Low serum interleukin-13 levels correlate with poorer prognoses for colorectal cancer patients. Int Surg 99(3): 223-229, 2014.

24 Anderson F, Downing GM, Hill J, Casorso L and Lerch N: Palliative performance scale (pps): A new tool. J Palliat Care 12(1): 5-11, 1996.

25 Jung KJ, Won D, Jeon C, Kim S, Kim TI, Jee SH and Beaty TH: A colorectal cancer prediction model using traditional and genetic risk scores in Koreans. BMC Genet 16: 49, 2015.

26 Ruopp MD, Perkins NJ, Whitcomb BW and Schisterman EF: Youden index and optimal cut-point estimated from observations affected by a lower limit of detection. Biom J 50(3): 419-430, 2008.

27 Pearce $\mathrm{J}$ and Ferrier S: Evaluating the predictive performance of habitat models developed using logistic regression. Ecological Modelling 133: 225-245, 2000.

28 Elith J and JR L: Predicting species distributions from museum and herbarium records using multiresponse models fitted with multivariate adaptive regression splines. Diversity Distributions 13: 265-275, 2007.

29 Kirk P, Kirk I and Kristjanson LJ: What do patients receiving palliative care for cancer and their families want to be told? A Canadian and Australian qualitative study. BMJ 328(7452): 1343, 2004.

30 Steinhauser KE, Christakis NA, Clipp EC, McNeilly M, McIntyre L and Tulsky JA: Factors considered important at the end of life by patients, family, physicians, and other care providers. JAMA 284(19): 2476-2482, 2000.

31 Morita T, Bito S, Koyama H, Uchitomi Y and Adachi I: Development of a national clinical guideline for artificial hydration therapy for terminally ill patients with cancer. J Palliat Med 10(3): 770-780, 2007.

32 Chiang JK, Lai NS, Wang MH, Chen SC and Kao YH: A proposed prognostic 7-day survival formula for patients with terminal cancer. BMC Public Health 9: 365, 2009.

33 Vigano A, Dorgan M, Buckingham J, Bruera E and SuarezAlmazor ME: Survival prediction in terminal cancer patients: A systematic review of the medical literature. Palliat Med 14(5): 363-374, 2000.

34 Bruera E, Miller MJ, Kuehn N, MacEachern T and Hanson J: Estimate of survival of patients admitted to a palliative care unit: A prospective study. J Pain Symptom Manage 7(2): 82-86, 1992.

Received November 8, 2016

Revised November 28, 2016

Accepted November 29, 2016 